Astronomical Journal, March 2001, Vol 121

\title{
Fractal Structure in Galactic Star Fields
}

\author{
Bruce G. Elmegreen ${ }^{1}$ and Debra Meloy Elmegreen ${ }^{2}$
}

\begin{abstract}
The fractal structure of star formation on large scales in disk galaxies is studied using the size distribution function of stellar aggregates in kpc-scale star fields. Achival HST images of 10 galaxies are Gaussian smoothed to define the aggregates, and a count of these aggregates versus smoothing scale gives the fractal dimension. Fractal and Poisson models confirm the procedure. The fractal dimension of star formation in all of the galaxies is $\sim 2.3$. This is the same as the fractal dimension of interstellar gas in the Milky Way and nearby galaxies, suggesting that star formation is a passive tracer of gas structure defined by self-gravity and turbulence. Dense clusters like the Pleiades form at the bottom of the hierarchy of structures, where the protostellar gas is densest. If most stars form in such clusters, then the fractal arises from the spatial distribution of their positions, giving dispersed star fields from continuous cluster disruption. Dense clusters should have an upper mass limit that increases with pressure, from $\sim 10^{3} \mathrm{M}_{\odot}$ in regions like the Solar neighborhood to $\sim 10^{6} \mathrm{M}_{\odot}$ in starbursts.
\end{abstract}

keywords: galaxies: star clusters — ISM: structure — stars: formation

\section{Introduction}

Interstellar gas is observed to have a fractal structure ranging from sub-parsec scales to $>10$ parsec scales in non-self-gravitating clouds (Crovisier \& Dickey 1983; Green 1993; Vogelaar \& Wakker 1994), from parsec to $\sim 100$ pc scales in self-gravitating clouds (Dickman, Horvath \& Margulis 1990; Scalo 1990; Falgarone, Phillips, \& Walker 1991; Elmegreen \& Falgarone 1996; Stutzki et al. 1998), and from 10 pc scales to $\sim 5$ kiloparsec scales in large sections of galaxies for both the stars (Feitzinger, \& Braunsfurth 1984; Feitzinger, \& Galinski 1987; Elmegreen \& Efremov 1996; Efremov \& Elmegreen 1998; Selman \& Melnick 2000; Elmegreen et al. 2001) and the gas (Stanimirovic et al. 1999; Westpfahl et al. 1999; Keel \& White 2000; Elmegreen, Kim, \& Staveley-Smith 2001). In many cases, the observed range of scales is probably a lower limit, because it begins at the scale of resolution of the instrument and ends at the size of the mapped region.

The observation of fractal structure in the gas suggests that stars should form in fractal patterns too if their birth places uniformly follow the densest regions (see review in Elmegreen et al. 2000). Here we show evidence for such fractal patterns in the star fields of other galaxies covering a range of scales from the resolution limit of $\sim 10$ parsecs to giant spiral arm complexes that are several kiloparsecs in length. Fractal models that are fit to these observations suggest that the fractal dimension of star formation is around 2.3, which is the same as for the interstellar gas.

\footnotetext{
${ }^{1}$ IBM Research Division, T.J. Watson Research Center, P.O. Box 218, Yorktown Heights, NY 10598, bge@watson.ibm.com

${ }^{2}$ Department of Physics and Astronomy, Vassar College, Poughkeepsie, NY 12604; e-mail: elmegreen@vassar.edu
} 


\section{Observations}

We studied optical images of 10 galaxies using Hubble Space Telescope archival data. The HST gives a relatively clear view of star fields in other galaxies because the factor of 10 improvement in angular resolution over ground-based images means that distant galaxies can be studied with the same spatial resolution as conventional images of nearby galaxies but with $\sim 100$ times fewer foreground stars.

The galaxies are listed in Table 1. HST archival images of big star forming regions in these galaxies were convolved with Gaussian point spread functions in order to blur them to varying degrees. The count of the number of optical clusters versus the smoothing scale was then plotted on a log-log plot and the slope was determined. For a fractal distribution, the slope of such a plot is the fractal dimension, $D$ (Mandelbrot 1983), provided there is no loss of counts from blending. The slope was determined for the five biggest star fields in NGC 2207, and for the biggest star fields in 8 other galaxies, with two fields in NGC 5457.

An example of this process is illustrated in Figure 1. This shows six stages in the smoothing of a 5 kpc-long star field in the south eastern arm of NGC 2207 (see Elmegreen et al. 2000). We count 75 separate centers for star formation (i.e., clusters) in the highest resolution image, and we count 52, 38, 21, 8, and 2 centers in the five other images, respectively, which were smoothed in successive steps equal to a factor of 2 in scale.

The cluster counts are shown on the left in Figure 2. The counts for five star fields in NGC 2207 are on the top left and the counts for 9 star fields in the 8 other galaxies are on the bottom left. The distribution function for the number, $n$, of clusters versus scale, $S$, is $n(S) d \log S \propto S^{-D} d \log S$ for $D=1.12 \pm 0.25$ in the 14 total cases. Thus the fractal dimension would be $D \sim 1.12 \pm 0.25$ without blending. However, the complexes overlap and blend with each other because of their hierarchical structure. Thus we have to model this counting process with images of known fractal dimensions in order to reconstruct the dimension of the real star fields.

\section{Models}

Fractal and other models of clusters were made by computer in order to fit the slope of the observed $n(S)$ relation, and to see whether we can tell the difference between a fractal pattern and completely random pattern, which has a Poisson distribution. Figure 3 shows sample models before Gaussian smoothing; on

Table 1: Galaxies studied

$\begin{array}{llcc}\text { Galaxy } & \text { type } & \text { Distance }(\mathrm{Mpc}) & \text { Image scale (pc per WF px) } \\ \mathrm{N} 2207 & \mathrm{SAB}(\mathrm{rs}) \mathrm{bc} & 35 & 17 \\ 2366 & \mathrm{IB}(\mathrm{s}) \mathrm{m} & 2.9 & 1.4 \\ 3184 & \mathrm{SAB}(\mathrm{rs}) \mathrm{cd} & 8.7 & 4.2 \\ 3423 & \mathrm{SA}(\mathrm{s}) \mathrm{cd} & 10.9 & 5.2 \\ 4051 & \mathrm{SAB}(\mathrm{rs}) \mathrm{bc} & 17 & 8.2 \\ 4303 & \mathrm{SAB}(\mathrm{rs}) \mathrm{bc} & 15.2 & 7.3 \\ 4449 & \mathrm{IBm} & 3 & 1.4 \\ 5068 & \mathrm{SB}(\mathrm{s}) \mathrm{d} & 6.7 & 3.2 \\ 5457 & \mathrm{SAB}(\mathrm{rs}) \mathrm{cd} 5.4 & 2.6 & \\ \mathrm{I} 2163 & \mathrm{SB}(\mathrm{rs}) \mathrm{c} \mathrm{pec} & 35 & 17\end{array}$


the left is a Poisson distribution of centers, in the middle is a fractal with $D=1.3$, and on the right is a fractal with $D=2.3$.

The Poisson pattern was made by placing 2048 points on an $(\mathrm{x}, \mathrm{y})$ plane with random positions $x$ and $y$ distributed uniformly between values 0 and 1 . This is a two-dimensional array, but is equivalent to a three dimensional array viewed in projection (i.e., random $z$ values collapsed to the same $z$ value). To simulate what we already know about clusters, the points were given finite sizes that have a power-law distribution function comparable to the observed power law for individual star-cluster sizes, namely $n(R) d \log R \propto R^{-2.3} d \log R$ (Elmegreen \& Salzer 1999; Elmegreen et al. 2001). In reality, this intrinsic distribution probably arises from the same fractal structure that we seek to measure in the distribution of cluster center positions, just as the size and mass distributions of individual molecular clouds display a microcosm of the same overall fractal structure that is seen on much larger scales in the distribution of interstellar gas (Elmegreen \& Falgarone 1996). However, the conventional picture has individual clouds or star-forming regions with a power law size distribution and a random distribution for the centers of these regions. Here we seek to disprove this conventional picture by showing that the center positions are fractal without commenting directly on the intrinsic size distribution.

The size distribution used for these models is consistent with the size-luminosity relation for star clusters, $L \propto R^{2.3}$ (Elmegreen et al. 2001), and with the luminosity distribution function for clusters and HII regions, $n(L) d L \sim L^{-2} d L$ (Kennicutt, Edgar, \& Hodge 1989; Battinelli et al. 1994; Elmegreen \& Efremov 1997; Comeron \& Torra 1996; Feinstein 1997; Oey \& Clarke 1998; McKee \& Williams 1997). We have commented previously how these relations are also consistent with a purely fractal distribution, the first giving the fractal dimension in another way (Pfenniger \& Combes 1994; Larson 1994; Elmegreen \& Falgarone 1996; Elmegreen et al. 2001), and the second coming from a hierarchical distribution with any fractal dimension (Fleck 1996; Elmegreen \& Falgarone 1996).

The model fractal distributions are generated by uniformly selecting some random number, $N_{1}$, in the range from 1 to $N$ and then using this for the number of star-forming regions in the first, or highest, level in the hierarchy of structures. The $(\mathrm{x}, \mathrm{y})$ positions of these $N_{1}$ regions are then determined uniformly in the interval of position from 0 to 1 using other random variables. Second, we go to the position of each of these $N_{1}$ regions and select other random numbers, $N_{2,1}, N_{2,2}, \ldots$, in the interval from 1 to $N$. These are the number of level-2 sub-regions associated with each previous region in level 1. For each level-2 subregion, we find new random positions, but this time separated from the level-1 positions by a random number in the interval from 0 to $L<1$, where $L=10^{\log (N) / D}$ for fractal dimension $D$. For the level-3 positions, we find the number of sublevels first in the same way, and then choose new positions around each, separated by a random number in the interval from 0 to $L^{2}$. With these successively smaller separations, we make clusters with a fractal dimension, $D=\log (N) / \log (L)$. This process is continued for 6 levels.

When the selection of fractal positions is finished, we assign each circle a size randomly distributed according to the function $n(R) d \log R \propto R^{-2.3} d \log R$, as discussed above. This is done by solving for $R$ in the equation

$$
R=\frac{R_{\min }}{\left\{1-\left(1-\left[R_{\min } / R_{\max }\right]^{2.3}\right) \xi\right\}^{1 / 2.3}}
$$

where $\xi$ is a random number uniformly distributed in the interval from 0 to 1 . An image of these circles is then stored on a $512 \times 512$ grid. The value of the image is set to 1 inside each circle, and when two or more circles overlap, the value in the image is the sum of each contribution. This procedure is consistent with the approximately constant surface brightness of star complexes that is implied by the luminosity-size relation given above. 
The model images are viewed in Photoshop with different Gaussian smoothing, stepped by factor-of-2 intervals from the original image. Thus the smoothing scales are $2,4,8,16,32$, and 64 pixels. The number of separate regions was counted by eye on each smoothed image.

Figure 2 shows the counts for each image as a function of smoothing scale. The Poisson maps are steeper than the fractals on these plots because there is less blending of the small features on the Poisson maps. This result illustrates the effects of projection mentioned by Mandelbrot (1983) and modeled with the shadows of crumpled newspapers by Beech (1992), namely, that the dimension of a projected fractal is approximately one less than the dimension of the full object. Figures with low fractal dimensions have the most blending and shallowest slopes. The average slopes for the Poisson, $D=2.3$, and $D=1.3$ models are $-1.72 \pm 0.04,-1.17 \pm 0.06$, and $-0.75 \pm 0.09$, respectively. The slope of the models is about equal to the slope of the observation for $D=2.3$.

\section{Discussion}

The distribution of star formation sites in a galaxy is a fractal with about the same dimension as the fractal interstellar gas. This implies that stars form from the gas, tracing its structure in a passive way. This result is not inconsistent with the observation that star formation occurs in the densest parts of the gas. We add to this observation only the fact that these densest parts are arranged in space on a fractal network. Presumably this distribution of star formation sites is the result of turbulence compression (Elmegreen 1994; Elmegreen 1999; Rosolowsky et al. 1999; MacLow \& Ossenkopf 2000; Pichardo et al. 2000) and gravity (Semelin, \& Combes 2000).

The fractal distribution of star formation sites is consistent with the observation that the total duration of star formation in a region is always around 2 crossing times, regardless of scale (Elmegreen 2000). It takes only $\sim 1$ crossing time for turbulence to establish the hierarchy of structures from an initially uniform gas, and it takes another crossing time on any level for all of the smaller scale processes, which operate faster, to make their stars.

Dense star clusters form at the bottom of this hierarchy of gas and star-formation structures, where the density is large. The maximum mass of a dense cluster depends on the local pressure and density as,

$$
M \leq 6 \times 10^{3}\left(P / 10^{8} \mathrm{~K} \mathrm{~cm}^{-3}\right)^{3 / 2}\left(n / 10^{5} \mathrm{~cm}^{-3}\right)^{-2} .
$$

This comes from the equations $P \sim 0.1 G M^{2} / R^{4}$ and $n \sim 3 M /\left(4 \pi \mu R^{3}\right)$ for cloud mass $M$, radius $R$, core pressure $P$, and mean molecular weight $\mu \sim 4 \times 10^{-24} \mathrm{~g}$ (Elmegreen 1989; Harris \& Pudritz 1994). A core pressure of $10^{8} \mathrm{~K} \mathrm{~cm}^{-3}$ and an average density of $\sim 10^{5} \mathrm{~cm}^{-3}$ are chosen for normalization because these are observed in the Orion regions where dense clusters form (Lada, Evans, \& Falgarone 1997). The pressure comes from the density multiplied by the square of the observed velocity dispersion of $\sim 1.5 \mathrm{~km} \mathrm{~s}^{-1}$. This density of $\sim 10^{5} \mathrm{~cm}^{-3}$ corresponds to $5.9 \times 10^{3} \mathrm{M}_{\odot} \mathrm{pc}^{-3}$, and to a final star density of $\sim 10^{4} \mathrm{stars} \mathrm{pc}^{-3}$ with $50 \%$ efficiency. This equation illustrates why the galactic or "open" clusters in our Milky Way disk, which are born with stellar densities like this, tend to be smaller than several thousand solar masses. Higher ambient interstellar pressures should lead to higher cloud core pressures and the formation of more massive clusters with the same and higher densities.

Most star formation seems to occur in dense clusters, although many of these may disperse soon after birth (Kroupa 2000). Even so, the distribution of young stars should still be fractal in an overall fractal gas because the velocity dispersion of each cluster is small compared to the turbulent velocity dispersion of the 
larger region around it. This means that the timescale for the larger scale in the hierarchy of structures is always shorter than the time for a dense cluster to expand to this large scale. Because of this, cluster

evaporation and dispersal on the small scale should not smear out the fractal pattern that is continuously established by turbulence and self-gravity on the large scale.

\section{REFERENCES}

Battinelli P., Brandimarti A. \& Capuzzo-Dolcetta R. 1994, A\&AS, 104, 379

Beech, M. 1992, ApSpS, 192, 103

Comerón, F., \& Torra, J. 1996, A\&A, 314, 776

Crovisier, J., \& Dickey, J.M. 1983, A\&A, 122, 282

Dickman, R. L., Horvath, M. A., \& Margulis, M. 1990, ApJ, 365, 586

Efremov, Y.N., \& Elmegreen, B.G. 1998, MNRAS, 299, 588

Elmegreen, B.G., 1989, ApJ, 338, 178

Elmegreen, B.G., 1999, ApJ, 527, 266

Elmegreen, B.G. 2000, ApJ, 530, 277

Elmegreen, B.G., \& Efremov, Yu. N. 1996, ApJ, 466, 802

Elmegreen, B.G., \& Falgarone, E. 1996, ApJ, 471, 816

Elmegreen, B.G., \& Efremov, Yu.N. 1997, ApJ, 480, 235

Elmegreen, B.G., Efremov, Y.N., Pudritz, R., \& Zinnecker, H. 2000, in Protostars and Planets IV, eds. V. G. Mannings, A. P. Boss, \& S. S. Russell, Tucson: Univ. Arizona Press, p. 179

Elmegreen, B.G., Kaufman, M., Struck, C., Elmegreen, D.M., Brinks, E., Thomasson, M., Klarić, M., Levay, Z., English, J., Frattare, L. M., Bond, H. E., Christian, C. A., Hamilton, F., Noll, K., 2000, AJ, 120,630

Elmegreen, B.G., Kim, S., \& Staveley-Smith, L. 2001, ApJ, in press

Falgarone, E., Phillips, T. G., \& Walker, C. K. 1991, ApJ, 378, 186

Elmegreen, D.M., \& Salzer, J.J. 1999, AJ, 117, 764

Elmegreen, D.M., Kaufman, M., Elmegreen, B.G., Brinks, E., Struck, C., Klaric, M., Thomasson, M. 2001, $\mathrm{AJ}$, in press

Feinstein, C. 1997, ApJS, 112, 29

Feitzinger, J.V., \& Braunsfurth, E. 1984, A\&A, 139, 104

Feitzinger, J.V., \& Galinski, T. 1987, A\&A, 179, 249

Fleck, R.C., Jr. 1996, ApJ, 458, 739

Green, D.A. 1993, MNRAS, 262, 327

Harris, W.E., \& Pudritz, R.E. 1994, ApJ, 429, 177

Keel, W.C., \& White, R.E. III 2000, in press

Kennicutt, R.C. 1998, ApJ, 498, 541

Kennicutt, R.C., Edgar, B.K., \& Hodge, P.W. 1989, ApJ, 337, 761 
Kroupa, P. 2000, in From Darkness to Light, eds. Montmerle, T., \& Andre, P., ASP Conf. Series, in press.

Lada, E. A., Evans, N. J., II, Depoy D. L., Gatley, I. 1991, ApJ, 371, 171

Lada, E.A., Evans, N.J., II., \& Falgarone, E. 1997, ApJ, 488, 286

Larson, R.B. 1994, in Clouds, Cores and Low Mass Stars, ed. D.P. Clemens \& R. Barvainis, ASP Conf. Ser. 65 , p. 125

MacLow, M.M., \& Ossenkopf, V. 2000, A\&A, 353, 339

Mandelbrot, B.B. 1983, The Fractal Geometry of Nature, (San Francisco: Freeman)

McKee, C.F., \& Williams, J.P. 1997, 476, 144

Oey, M. S., \& Clarke, C. J. 1998, AJ, 115, 1543

Pfenniger, D. \& Combes, F. 1994, A\&A, 285, 94

Pichardo, B., Vázquez-Semadeni, E., Gazol, A., Passot, T., Ballesteros-Paredes, J. 2000, ApJ, 532, 353

Rosolowsky, E.W., Goodman, A.A., Wilner, D.J., \& Williams, J.P. 1999, ApJ, 524, 887

Scalo, J. 1990, in Physical Processes in Fragmentation and Star Formation, eds. R. Capuzzo-Dolcetta, C. Chiosi, \& A. Di Fazio, Dordrecht: Kluwer, p. 151

Selman, F.J., \& Melnick, J. 2000, ApJ, 534, 703

Semelin, B., \& Combes, F. 2000, A\&A, 360, 1096

Stanimirovic, S., Staveley-Smith, L., Dickey, J.M., Sault, R.J., \& Snowden, S.L. 1999, MNRAS, 302, 417

Stutzki, J., Bensch, F., Heithausen, A., Ossenkopf, V., \& Zielinsky, M. 1998, A\&A, 336, 697

Vogelaar, M. G. R., \& Wakker, B. P. 1994, A\&A, 291, 557

Westpfahl, D.J., Coleman, P.H., Alexander, J., Tongue, T. 1999, AJ, 117, 868

Whitmore, B.C., Zhang, Q., Leitherer, C., Fall, S.M., Schweizer, F., Miller, B.W. 1999, AJ, 118, 1551 
Fig. 1.- (see jpg file) Six levels of Gaussian smoothing of a star-forming patch in the galaxy NGC 2207. The number of pixels in the Gaussian smoothing function is shown in each panel. The number of objects is plotted as a function of this smoothing length in Fig. 2.

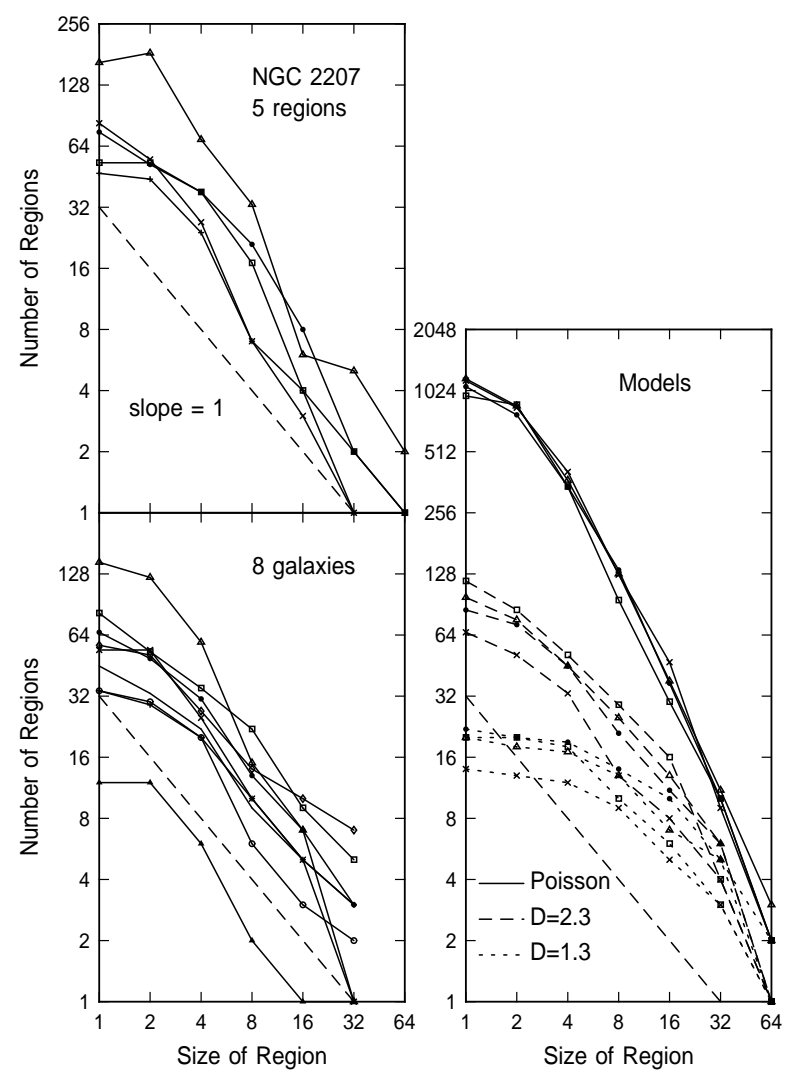

Fig. 2.- The number of star-forming concentrations is plotted as a function of the smoothing scale for five regions in NGC 2207 on the top left, for 9 regions in 8 galaxies on the lower left, and for three types of models on the right. The dashed line has a slope of -1 on this $\log -\log$ plot. The observations are best fit by a fractal with a dimension of 2.3 , as shown by the dashed line on the right.

Fig. 3.- (see jpg file) Three models for the spatial distribution of star-forming regions. The sizes of the regions have the observed power law distribution. These models are Gaussian smoothed to varying degrees to make the counts shown on the right in Fig. 2. 
This figure "elmegreen.fig1.jpg" is available in "jpg" format from: http://arxiv.org/ps/astro-ph/0012184v1 
This figure "elmegreen.fig3.jpg" is available in "jpg" format from: http://arxiv.org/ps/astro-ph/0012184v1 